\title{
A NEW KIND OF BEAM PIPE FOR RAPID CYCLING PROTON SYNCHROTRONS
}

\author{
Zhijing Tang*, Weiren Chou*, Alex Chen*, FNAL, Batavia, IL 60510, USA
}

\begin{abstract}
A thin metallic beam pipe reinforced by multi-layer spiral metallic ribs is proposed for rapid cycling proton synchrotrons. The pipe is made of Inconel 718 with thickness of a few tenths of $\mathrm{mm}$. Each spiral rib has a cross section of about $0.3 \mathrm{~mm}^{2}$ and can be bonded to the pipe by using laser deposition technique (e.g., precision metal deposition, or PMD). Compared with other designs (e.g.., ceramic beam pipe with a metallic cage used in the ISIS at the RAL), this new pipe will reduce the magnet aperture significantly, which, in turn, reduces the construction and operating cost of a synchrotron. Numerical simulations and analytical modeling are used to investigate the structural strength and deformation, and the eddy current effects, including heating, magnetic field distortion and the electro-magnetic force on the beam pipe. The results show that this new beam pipe will work. It can be employed to high intensity rapid cycling proton synchrotrons, such as the proton driver at FERMILAB and the JHF at JAERI/KEK. Effort to build a prototype is under way.
\end{abstract}

\section{THE BEAM PIPE}

The cross section of the proposed beam pipe is 6 inch by 4 inch oval with thickness of 0.008 inch. The material is Inconel 718. The laser precision metal deposition (PMD) method will be used to put a spiral rib (same material with 18 mils height and 28 mils width can be applied repeatedly) around the tube to increase its strength against buckling. We select 10 layers of reinforcement (hence the total height of the rib is $0.18 \mathrm{inch}$ ) and the pitch of the rib is set to $1 \mathrm{inch}$. Some relevant material properties of Inconel 718 are: tension modules $E=29 \mathrm{e} 6$ psi, yield strength $\sigma_{\mathrm{y}}=171 \mathrm{e} 3 \mathrm{psi}$, ultimate strength $\sigma_{\mathrm{u}}=$ $196 \mathrm{e} 3 \mathrm{psi}$, electric resistivity $\lambda=125 \mathrm{e}-8 \mathrm{ohm}-\mathrm{m}$.

The beam pipe is subject to some loads. Mechanically there is a vacuum pressure, and electro-magnetically there is a time varying magnetic field:

$$
B^{\text {ext }}=0.85-0.632 \cos (\omega t)+0.079 \sin (2 \omega t),
$$

where $\omega=2 \pi f$, and $f=15 \mathrm{~Hz}$. This magnetic field is perpendicular to the major axis of the beam pipe cross section.

* Work supported by the US Department of Energy under contract No. DE-AC02-76CH03000.
Vacuum pressure is simple to analyze, but the effects of the time vary magnetic field is complex. We have to consider the eddy current, eddy current induced magnetic field and power loss, and electro-magnetic force.

\section{STRUCTURAL MODEL}

For structural analysis, we made a six inch finite element model. The only load considered here is the vacuum pressure of 14.5 psi. Electro-magnetic force will be discussed later. The boundary conditions are: $u_{\mathrm{Z}}=0$ at $z=0 ; u_{\mathrm{X}}=0$ at $x=0$ and $z=0 ; u_{\mathrm{y}}=0$ at $y=0$ and $z=0$.

Fig. 1 shows the maximum equivalent stress of 144 ksi. This maximum occurs near the end. If we look at the middle section of the model, which is more typical, we have maximum stress of $130 \mathrm{ksi}$. So we take $130 \mathrm{ksi}$ as the maximum stress in the vacuum tube. Check the maximum stress against the ASME pressure vessel code [1], which gives the limiting stress about $170 \mathrm{ksi}$, we found out that this number is $\mathrm{OK}$.

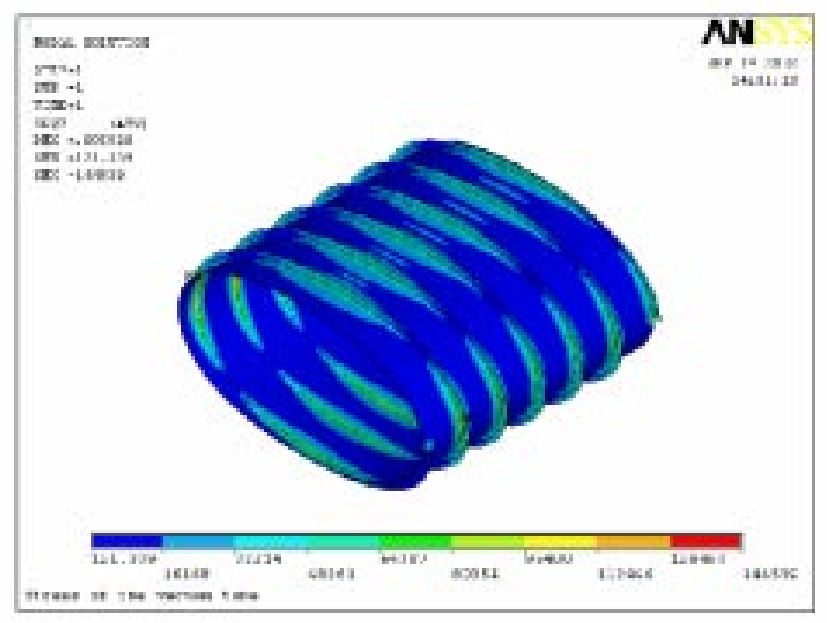

Figure 1. FEA structural model, stress plot

Fig. 2 shows the deformation of the beam pipe under vacuum pressure. For clarity, reinforce rib is not plotted. The major radius extends 0.055 inch $(1.40 \mathrm{~mm})$, and the minor radius shrink 0.089 inch $(2.26 \mathrm{~mm})$.

From these results, we see that the proposed beam pipe will survive the vacuum pressure. The only thing not so perfect is that the deformation is a little too big. To achieve required tube shape under vacuum condition, the 
beam pipe initially can be made with smaller major radius and larger minor radius.

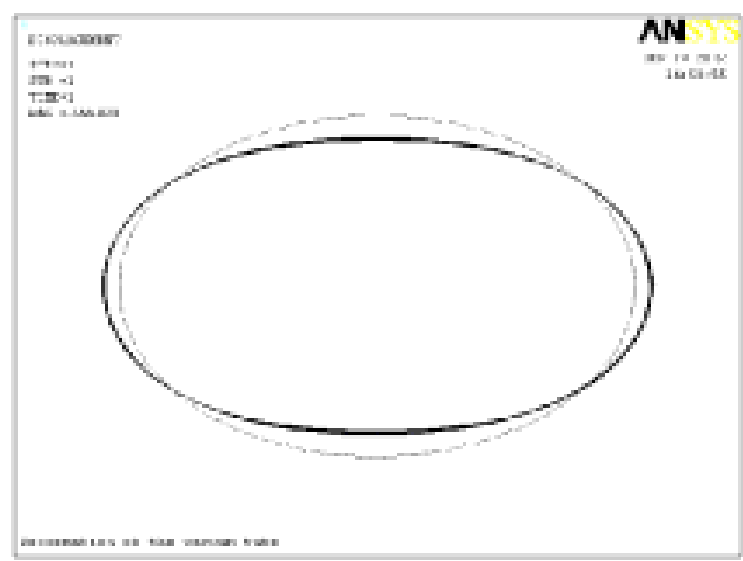

Figure 2. Deformation of Beam Pipe

\section{ELECTRO-MAGNETIC EFFECTS}

Since the beam pipe is made of metal, time varying external magnetic field will induce eddy current. This eddy current will have two effects: distort the external field and cause power loss.

For a single spiral rib, an argument based on symmetry will lead to the conclusion that the eddy current caused by the spiral rib is negligible compared with the beam pipe itself [2]. Once we have this conclusion, the analysis of the eddy current effects is easy. For oval beam pipe of infinite length, analytic solution can be found [2]. However, for completeness, we made a finite element model, and did some harmonic analysis. The results are compared with the analytic results.

For electro-magnetic analysis, we model only the beam pipe without the reinforce rib. Use 3-d element, we made a quarter model. The model length is 1 inch. The inside and outside are filled with vacuum and air elements. Since the effect is linear, we can analyze the $15 \mathrm{~Hz}$ field and 30 $\mathrm{Hz}$ field separately. The constant field has no eddy current effect.

For $15 \mathrm{~Hz}$ field, $B_{1}=0.632 \mathrm{~T}$, calculated power loss is $1.957 \mathrm{w}$, for $30 \mathrm{~Hz}$ field, $B_{2}=0.632 / 8 \mathrm{~T}$, power loss is $0.122 \mathrm{w}$. Add together, we have $2.079 \mathrm{w}$ power loss for our model. Remember our model is a quarter model, so the total power loss should be $8.316 \mathrm{w} / \mathrm{in}$, or $327 \mathrm{w} / \mathrm{m}$. This number compare well with the analytical result, which is $323 \mathrm{w} / \mathrm{m}$.

Now consider the magnetic field inside the vacuum tube on $y=0$ plane. The results show that the magnitudes are almost exactly the same as that of the applied external fields, and the lag angles are extremely small. That is to say, that the magnetic field distortion due to eddy current is extremely small. Therefore, no correction will be needed. The analytic results are: The ratio of magnetic field induced by the eddy current to the external field is $0.613 \mathrm{e}-3$ for $15 \mathrm{~Hz}$ field and $1.227 \mathrm{e}-3$ for $30 \mathrm{~Hz}$ field.

The electro-magnetic force is calculated analytically. It is a time changing quantity. We integrate the total force acting on $x>0$ half beam pipe

$$
F=1.462 B \dot{B}
$$

The Maximum value of this function is $110 \mathrm{~N} / \mathrm{m}$. Compare this with the total force caused by the vacuum pressure, which is $10157 \mathrm{~N} / \mathrm{m}$. We have the result that the former is about $1 \%$ of the later. Therefore when we did the structural analysis by neglecting the magnetic force, the error will be about $1 \%$.

\section{CONCLUSIONS}

In summary, we restate the main results of the analysis in following: 1) The maximum stress in beam pipe under vacuum pressure is $144 \mathrm{ksi}$. 2) The deformation extends major radius $1.40 \mathrm{~mm}$, and shrinks the minor radius 2.26 $\mathrm{mm} .3$ ) The power loss under considered magnetic field is $327 \mathrm{w} / \mathrm{m}$. 4) The eddy current induced magnetic field can be neglected compared with the external field. 5) The electro-magnetic force can be neglected compared with the vacuum pressure.

From this study, we know that the proposed beam pipe should work, at least in theory. The problems will occur during manufacturing process, there the imperfections will be introduced. Some degree of damage to the tube material will be unavoidable. Since this tube is so thin, the final strength of the beam pipe will completely depend on the perfection of the manufacture techniques, therefore prototype study is needed.

\section{REFERENCE}

[1] ASME, Pressure Vessel Code (1995), Section VIII, Division 2.

[2] Z. Tang, A New Kind of Vacuum Tube for Proton Driver (2002), FERMILAB-TM-2118 